DOI: 10.12731/2070-7568-2017-3-164-176

УДК 330.3

\title{
ПРОГНОЗИРОВАНИЕ ЦЕЛЕВЫХ ПОКАЗАТЕЛЕЙ ГОСУДАРСТВЕННОГО ОБОРОННОГО ЗАКАЗА
}

\section{Смуров А.M.}

Формирование и реализачия государственного оборонного заказа в настоящее время является актуальной задачей. Эффективность её решения находится в прямой зависимости от степени научно-методического сопровождения соответствующих мероприятий, одним их которых является проиесс прогнозирования целевых показателей государственного оборонного заказа.

Цель статьи - разработка методики прогнозирования количественных значений цуелевых показателей государственного оборонного заказа.

Используемые методы исследования: метод анализа и синтеза, экономико-математическое моделирование

Основные результаты: построена система целевых показателей государственного оборонного заказа, разработана методика прогнозирования их количественных значений, выявлено место государственного оборонного заказа в системе обеспечения национальной безопасности государства

Ключевые слова: военная организащия государства; государственный оборонный заказ; прогнозирование; целевые показатели.

\section{FORECAST OF GOAL INDEXES OF THE STATE DEFENSE ORDER}

\section{Smurov A.M.}

Preparation and execution of the state defense order is now an important task. Effectiveness of its solution directly depends on the level of methodological basis of measures necessary to carry out this order. 
One of these measure is the process of determination of goal indexes of the state defense order.

Goal of the paper - working out a method of determination of goal indexes of the state defense order.

Methods of research - analysis and synthesis, economical and mathematical modelling

Main results: system of goal indexes of the state defense order, method of forecast of their quantitative values, place of the state defense order in the national security of the state

Keywords: military organization of the state; state defense order; forecasting; goal indexes.

В настоящее время большое значение имеет повышение эффективности механизма государственного оборонного заказа (ГО3) [4, 5, $9,10,11]$. Это связано, с одной стороны, со снижением доходов государственного бюджета (из-за падения цен на нефть и общей неблагополучной ситуации в экономике), а с другой стороны - с ростом геополитических угроз [3]. В совокупности это предъявляет повышенные требования к эффективности системы распределения финансов между различными направлениями государственного заказа.

В оптимизации нуждаются все элементы механизма реализации ГОЗ:

- обоснование перечня направлений финансирования;

- определение целевых показателей ГОЗ;

- процедура отбора исполнителей ГОЗ;

- алгоритм выстраивания взаимодействия между военным заказчиком и исполнителем по ГОЗ и т. д.

Разумеется, в рамках одной статьи охватить все эти направления совершенствования ГОЗ невозможно, поэтому мы сосредоточимся на процедуре определения целевых показателей ГОЗ. Очевидно, что эта задача включает в себя две подзадачи.

Первая - построение системы целевых показателей ГОЗ. Формирование такой системы необходимо по причине того, что ГОЗ представляет собой комплексное, многоаспектное военно-экономическое явление, которое невозможно описать при помощи какого-либо одного показателя. Более того, его нельзя описать на ос- 
нове набора однородных (т. е. характеризующих отдельный аспект ГО3) показателей - такое описание неизбежно будет однобоким, и неизбежно приведет к неправильной постановке целей при формировании ГОЗ, и, как следствие, к тому, что результат выполнения ГОЗ не сможет соответствовать реальным задачам обеспечения национальной безопасности. Именно по этой причине необходимо построить систему показателей, адекватно описывающую ГОЗ.

Вторая - разработка методики определения количественных значений показателей различных групп.

По нашему мнению, показатели ГОЗ должны разбиваться на три группы (мы не конкретизируем отдельные показатели, поскольку они, весьма вероятно, будут весьма многочисленны; мы хотим лишь показать принципиальную структуру системы показателей ГОЗ):

1) целевые показатели ввода в строй вооружения, военной и специальной техники (BВСТ). Эти показатели описывают то количество ВВСТ (по типам), которое должно быть введено в строй в течение определенного периода. Определение количественных значений показателей этой группы основывается на приоритетах развития видов и родов войск, которые, в свою очередь, формируются исходя из существующего и предполагаемого уровня военных угроз, а также на основе военной доктрины Российской Федерации;

2) целевые показатели финансирования. Эти показатели характеризуют тот объем финансирования, который должен быть выделен в течение соответствующего периода для того, чтобы обеспечить достижение целевых значений показателей ввода ВВСТ. Между показателями первой и второй группы существует сложная система отношений. С одной стороны, количественные значения показателей второй группы формируются исходя из показателей первой группы (поскольку финансирование выделяется для целей ввода в строй ВВСТ). Но, с другой стороны, бюджетные ограничения оказывают существенное влияние на объемы ввода ВВСТ. Фактически речь идет о непрерывном поиске компромисса между потребностями ВС РФ (обусловленными необходимостью обеспечивать национальную безопасность нашей страны военными методами) и бюджетными ограничениями. Иными словами, ни показатели первой группы, ни показатели второй группы нельзя признать первичными - их количественные значения взаимообусловлены; 
3) целевые показатели результативности. Речь идет о минимально допустимом уровне достижения значений целевых показателей двух первых групп (или, иначе, о пессимистическом сценарии реализации процессов финансирования и ввода ВВСТ). Эти показатели необходимы для контроля качества финансирования и ввода ВВСТ. Для показателей первой группы минимальный уровень результативности обуславливается минимально необходимым уровнем боеспособности ВС РФ, тогда как показатели результативности для второй группы задают минимальные целевые ориентиры при формировании государственного бюджета.

Стоит также отметить, что ГОЗ служит мощным инструментом регулирования экономической активности $[1,2]$, и поэтому, по нашему мнению, целесообразно ввести показатели, которые бы описывали целевые значения характеристик, описывающих деятельность предприятий, участвующих в выполнении ГОЗ (например, темпы их технического перевооружения, которые могут быть достигнуты благодаря финансированию, полученному предприятием благодаря участию в ГОЗ, или уровень импортозамещения, или степень локализации производственных цепочек, задействованных в процессе выполнения ГОЗ [8], и т. д.), а также социально-экономические параметры, характеризующие регион, в котором расположены эти предприятия (это означает, что ГОЗ должен содействовать росту экономического потенциала региона и улучшению его социальных показателей $[12,13])$. По сути дела, использование таких вторичных показателей позволяет использовать госзаказ не только как самоцель, но и как инструмент для развития национальной экономики. В текущих условиях затянувшегося экономического кризиса потребность в использовании госзаказа как инструмента стимулирования национальной экономики особенно высока.

Для показателей, характеризующих деятельность предприятий, участвующих в выполнении ГОЗ, можно также установить минимальные значения результативности (очевидно, для региональных социальноэкономических показателей введение результативности невозможно, поскольку эти показатели находятся вне контроля как предприятий, так и региональных властей). Это создаст дополнительные стимулы для этих предприятий к совершенствованию своей деятельности. Если эти 
пороговые значения не достигаются, то к предприятию могут применяться различные санкции вплоть до его исключения из системы ГОЗ.

Таким образом, можно утверждать, что целевые показатели ГОЗ делятся на две категории:

1) прямые (или первичные) показатели, характеризующие выполнение ГОЗ;

2) косвенные (или вторичные) показатели, позволяющие оценить вклад ГОЗ в развитие национальной экономики. В рамках данного исследования задача формирования количественных значений косвенных показателей рассматриваться не будет в силу её чрезвычайной трудоемкости.

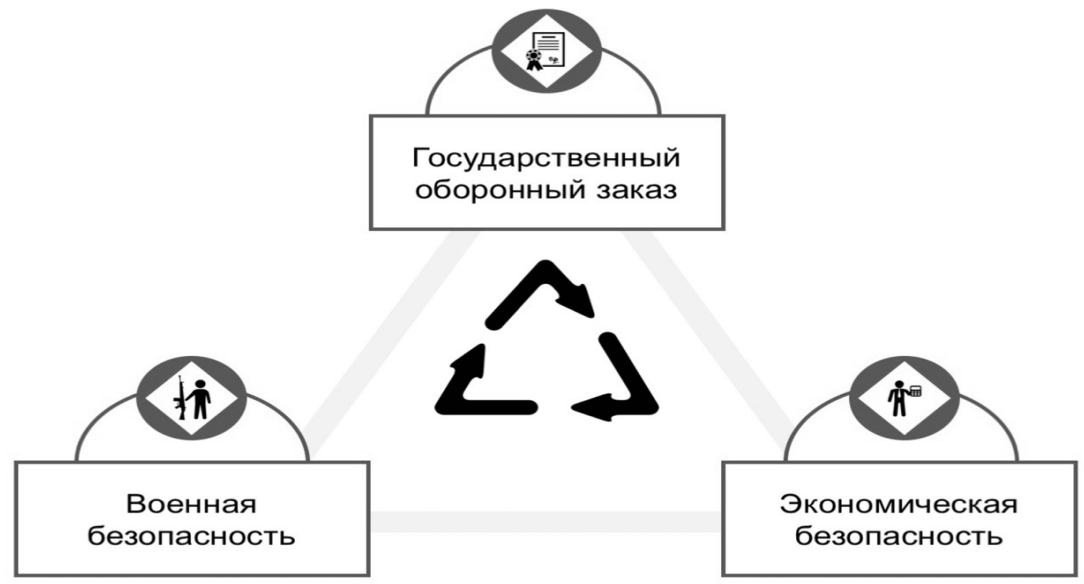

Рис. 1. Функции ГОЗ как инструмента обеспечения национальной безопасности государства

Источник: составлено автором.

Мы считаем принципиально важным использовать, при формировании ГОЗ, показатели как первой, так и второй группы. Отказ от использования показателей второй категории приведет к отрыву ГОЗ от потребностей национальной экономики, что, в конечном счете, скажется на национальной безопасности. Иными словами, ГОЗ должен быть направлен на формирование способности народного хозяйства своими силами обеспечить выполнение критических элементов оборонного заказа (по которым зависимость от иностранных поставщиков 
недопустима). Речь идет о развитии в стране собственных производственных мощностей, человеческого капитала, центров компетенций и поддерживающей инфраструктуры, за счет которых будет достигаться выполнением этих критических элементов ГОЗ. Стабильные денежные потоки, которые ГОЗ генерирует в интересах исполнителей, должны выступать одновременно источником и стимулом для исполнителей к инвестициям в создание перечисленных выше активов.

С учётом изложенного, совершенно очевидно, что ГОЗ направлен на обеспечение национальной безопасности государства на двух уровнях (см. рис. 1):

1) обеспечение военной безопасности государства путем снабжения ВС РФ необходимым для адекватного противостояния внешним военным угрозам ВВСТ. Это традиционная и очевидная функция ГОЗ;

2) обеспечение экономической безопасности государства за счет формирования способности народного хозяйства своими силами, независимо от внешних источников поставок, снабжать национальную экономику и военную организацию государства необходимыми для их полноценного функционирования ресурсами. Фактически это означает, что при размещении ГОЗ простая экономическая (учетная) логика, в соответствии с которой заказ нужно предлагать тому поставщику, который предложит наиболее выгодные условия конкретной поставки, применяться не должна (как это происходило в России на рубеже 2010-х. гг., когда заказы на поставки, например, вертолетонесущих десантных кораблей размещались на иностранных верфях, которые впоследствии, в силу политических причин, отказались от выполнения своих обязательств перед российским заказчиком). Предпочтение должно отдаваться отечественным поставщикам (или, как минимум, поставщикам из стран ЕврАзЭС), и при этом на них должны налагаться жесткие требования по уровню локализации производства конечной продукции по всей цепочке. Сотрудничество с иностранными контрагентами возможно только при условии их обязательства передачи критичных технологий российским предприятиям и содействия по организации выпуска конечной продукции на производственных мощностях, расположенных в России.

При этом, разумеется, не следует придавать показателям второй категории избыточного значения, поскольку в противном случае 
ГОЗ будет ориентирован на решение проблем национальной экономики, а не на достижение целей военной организации государства $[6,7]$. На предотвращение именно этого риска направлено требование президента РФ В.В. Путина о достижении определённого объема гражданской продукции в общем объеме выпуска предприятий ОПК (это создает стимулы для данных предприятий в своей деятельности не всецело ориентироваться на ГОЗ, а стремиться к независимой коммерциализации результатов своей деятельности).

С учётом очевидной важности задачи прогнозирования количественных значений целевых показателей ГОЗ, можно утверждать, что существующие подходы к её решению нуждаются в дальнейшем развитии [14]. Методика определения количественных значений показателей будет в первую очередь опираться на прогнозируемый уровень военных угроз. Пусть, в соответствии с выводами экспертов, речь идет о возможности реализации в течение рассматриваемого периода $m$ различных сценариев военных угроз, каждый из которых характеризуется определенной вероятностью наступления $p_{i}(i \in(1 ; 2 \ldots$; $m)$ ), значение которой определяется на основе экспертных оценок. Далее, военная безопасность Российской Федерации в случае реализации любого из этих сценариев может быть обеспечена $q_{i}$ способами (различающимися уровнем вовлеченности военной организации государства, и, как следствие, количественным и качественным составом задействованной военной техники). Каждый из этих способов требует для своей реализации затрат в размере $R_{l i}\left(l \in\left(1 ; 2 ; \ldots ; q_{i}\right)\right)$ и определяется своим набором ВВСТ $W_{l i}$ (для простоты и наглядности мы опускаем здесь тот факт, что эти наборы могут состоять из значительного количества видов ВВСТ, которые могут варьировать от стрелкового оружия до тяжелых авианесущих крейсеров; иными словами $l$-й набор для $i$-го сценария состоит из $G_{l i}$ видов элементов, и количество элементов каждого вида равно $\left.g_{k l i}, k \in 1 ; 2 ; \ldots G_{l}\right)$. Важно отметить, что элементы этих наборов $W_{l i}$ могут частично совпадать, т. е. совокупное число видов элементов $Q$, которые могут быть задействованы для противодействия военным угрозам, равно

$$
Q=\bigcup_{i=1}^{m} \bigcup_{l=1}^{q_{i}} G_{l i} .
$$


Кроме того, каждый из этих способов описывается соответствующим предполагаемым значением военной эффективности $M E_{l i}$.

Для прогнозного периода также определяется число сценариев $n$ наполнения доходной части бюджета, на основе которых для каждого сценария устанавливается величина денежных средств $D$ $(j \in(1 ; 2 ; \ldots ; n))$. Также для каждого сценария устанавливается (путем экспертных оценок) вероятность его реализации $p_{j}$. Это позволяет определить наиболее вероятное значение затрат государственного бюджета $M D$ :

$$
M D=\sum_{j=1}^{n} p_{j} D_{j}
$$

Величина $M D$ может быть использована как целевое значение показателя затрат государственного бюджета на выполнение ГОЗ (иными словами, целевое значение затрат должно стремиться к наиболее вероятному). Минимальное значение показателя затрат на ГО3 (т. е., в соответствии со сформулированным выше подходом, целевой показатель результативности) в первом приближении может быть определено как минимальное значение затрат $\min D_{j}$ из всех рассмотренных сценариев, однако такой подход представляется слишком прямолинейным и создает стимулы к минимизации (возможно, избыточной) затрат бюджета на закупки ВВСТ. Действительно, если минимально достаточными для ГОЗ будут признаны минимально возможные затраты на него, то, в условиях текущего экономического кризиса, у органов исполнительной власти будет соблазн ограничиться именно такой величиной затрат, что может создать риски недофинансирования ГОЗ и привести к угрозам для национальной безопасности государства.

На наш взгляд, можно выбрать альтернативный подход. Пусть $P$ - наиболее вероятное значение уровня военных угроз, т. е., в соответствии с принятыми выше обозначениями, $P$ - это тот уровень военных угроз, для которого выполняется условия $\max \left(p_{i}\right)$. Значение $P$ следует нормировать от 0 до 1 , где нулю соответствует полное отсутствие военных угроз, а 1 - максимальный уровень. 
Тогда минимальное допустимое значение затрат государственного бюджета $D_{\min }$ можно будет рассчитать по формуле:

$$
D_{\text {min }}=\min \left(D_{j}\right)+P\left(M D-\min \left(D_{j}\right)\right) \text {. }
$$

В этом случае минимальное допустимое значение затрат на ГОЗ не является фиксированным, а привязано к уровню военных угроз. При минимальном уровне военных угроз государство может ограничиться затратами, равными min $D_{j}$, тогда как при максимальном уровне военных угроз затраты должны быть не меньше наиболее вероятных $M D$.

Вернемся к задаче определения количественных значений целевых показателей ГОЗ по видам ВВСТ. Для этого мы сначала для каждого $i$-го сценария определим оптимальный набор ВВСТ $G_{i}^{\text {opt }}$ по следующему критерию:

$$
\left\{\begin{array}{c}
M E_{l i} \rightarrow \max \\
R_{l i} \leq M D .
\end{array}\right.
$$

Затем мы составим совокупный оптимальный набор ВВСТ $G^{*}$ (который представляет собой сумму оптимальных наборов ВВСТ, определенных для каждого сценария реализации военных угроз):

$$
G^{*}=\sum_{i=1}^{m} G_{i}^{o p t}
$$

Поскольку каждый $i$-й сценарий, в соответствии с прогнозами экспертов, реализуется с вероятностью $p_{i}$, мы скорректируем оптимальный набор ВВСТ (который должен войти в ГОЗ) на вероятности реализации сценариев:

$$
G_{\text {corr }}^{*}=\sum_{i=1}^{m} p_{i} G_{i}^{o p t}
$$

Далее нам необходимо перейти от информации о наборах ВВСТ к конкретным количественным значениям совокупных объемов ГОЗ по каждому $r$-му виду ВВСТ $Z_{r}^{t o t}(r \in 1 ; 2 ; \ldots ; Q$, где $Q$ - число видов ВВСТ, которое определяется по формуле (1)):

$$
Z_{r}^{\text {tot }}=\operatorname{round} \sum_{i=1}^{m} p_{i} Z_{r i} \text {, }
$$

где

round $(x)$ - функция округления (для того, чтобы не допустить нецелые значения количества единиц ВВСТ $r$-го вида); 
$Z_{r i}$ - количество единиц $r$-го вида ВВСТ, необходимого для $i$-го оптимального набора ВВСТ $G_{i}^{o p t}$.

Для определения минимально допустимой величины целевого значения показателя ГОЗ по $r$-му виду ВВСТ $Z_{r}^{\mathrm{min}}$ (т. е. целевой показатель результативности по объему закупок) используется аналогичный подход, однако условие (4) заменяется другим условием:

$$
\left\{\begin{array}{c}
M E_{l i} \rightarrow \max ; \\
R_{l i} \leq D_{\min } .
\end{array}\right.
$$

Иными словами, оптимальный объем закупок в этом случае привязывается не к наиболее вероятным, а к минимально допустимым значениям затрат государственного бюджета. Дальнейшие расчеты проводятся по формулам (5-7).

Подводя итог, мы можем констатировать, что:

- целевые показатели ГОЗ формируют сложную многоуровневую систему, каждый уровень которой необходим для достижения определенных целей ГОЗ. В частности, в них можно выделить прямые (ориентированные непосредственно на ГОЗ) и косвенные (предназначенные для обеспечения социально-экономического и народнохозяйственного развития государства) показатели. В свою очередь, прямые показатели включают в себя целевые показатели закупок, целевые показатели затрат государственного бюджета и целевые показатели результативности;

- прогнозирование значений целевых показателей ГОЗ опирается на предварительное определение прогнозных уровней военных угроз (и возможных вариантов противодействия им, при этом для каждого варианта экспертно устанавливается его военная эффективность) и прогнозной наполняемости государственного бюджета;

- цель прогнозирования целевых показателей ГОЗ заключается в обеспечении максимальной военной эффективности ВС РФ при непревышении целевых показателей затрат государственного бюджета на выполнение ГОЗ. 


\section{Список литературы}

1. Вертакова Ю.В., Положенцева Ю.С., Непочатых О.Ю. Методика выбора предприятий-реципиентов бюджетной поддержки при реализации государственной промышленной политики // Вестник Забайкальского государственного университета. 2017. Т. 23. № 2. C. $106-118$.

2. Вертакова Ю.В., Плотников В.А., Хорьков А.В., Лю Ч. Управление структурными преобразованиями экономических систем с учетом фактора риска // Известия Юго-Западного государственного университета. 2017. № 1 (70). С. 126-137.

3. Викулов С.Ф., Хрусталёв Е.Ю. Методология оценки оборонного и военно-экономического потенциалов государства // Национальные интересы: приоритеты и безопасность. 2013. № 29. С. 2-11.

4. Ерасова Е.А., Плотников В.А. Оборонно-промышленный комплекс России и перспективы его развития в условиях экономических санкций и реализации политики импортозамещения // Экономическое возрождение России. 2015. № 2. С. 102-110.

5. Князьнеделин Р.А., Смуров А.М. Проблемы организации эффективной системы управления государственными и корпоративными закупками // Проблемы экономики и управления нефтегазовым комплексом. 2016. № 6. С. 37-42.

6. Князьнеделин Р.А. Государственный заказ: теория, механизм исполнения, специфика реализации в оборонной сфере: монография / Князьнеделин Р.А., Наружный В.Е., Смуров А.М.; под редакцией д-ра экон. наук, проф. Плотникова В. А. СПб.: Изд-во СПбГЭУ, 2017. 159 с.

7. Козин М.Н., Алексеев А.В., Хачатрян Г.А. Эволюция нормативноправового регулирования государственных закупок: проблемы и противоречия // Вестник Саратовской государственной юридической академии. 2009. № 6 (70). С. 88-91.

8. Котляров И.Д. Локализация производства как инструмент импортозамещения // ЭКО. 2016. № 8. С. 128-140.

9. Курбанов А.Х., Наружный В.Е. Перспективы реализации программы импортозамещения в интересах оборонно-промышленного комплекса России в современных условиях // Проблемы современной экономики. 2015. № 3. С. 72-77. 
10.Курбанов А.Х., Наружный В.Е., Плотников В.А. Банковское сопровождение государственного оборонного заказа: специфика и особенности реализации // Управленческое консультирование. 2015. № 10. С. 101-109.

11. Курбанов А.Х., Плотников В.А. Направления развития институционального регулирования государственных закупок // Известия ЮгоЗападного государственного университета. 2011. № 2. С. 22-30.

12. Курбанов А.Х., Плотников В.А. Экономический потенциал страны как экономическая категория // Экономическое возрождение России. 2016. № 3. С. 45-56.

13. Сафиханов А.А., Козин М.Н., Курбанов Т.Х. Алгоритм управления отношениями военного заказчика и гражданского транспортного оператора в интересах развития системы материально-технического обеспечения военных потребителей в Арктике // Экономика и менеджмент систем управления. 2016. Т. 3.1. № 21. С. 170-176.

14. Семенихин А.Л. Методология военно-экономического прогнозирования системы развития материального обеспечения: Дисс. ... д-ра воен. наук. СПб.: ВАТТ, 1998. 264 с.

\section{References}

1. Vertakova Yu.V., Polozhentseva Yu.S., Nepochatykh O.Yu. Vestnik Zabaykalskogo gosudarstvennogo universiteta, 2017, V. 23, no 2, pp. 106-118.

2. Vertakova Yu.V., Plotnikov V.A., Khorkov A.V., Lyu Ch. Izvestiya Yugo-Zapadnogo gosudarstvennogo universiteta, 2017, no 1 (70), pp. 126-137.

3. Vikulov S.F., Khrustalev E.Yu. Natsionalnye interesy: prioritety i bezopasnost, 2013, no 29, pp. 2-11.

4. Yerasova E.A., Plotnikov V.A. Ekonomicheskoe vozrozhdenie Rossii, 2015, no 2, pp. 102-110.

5. Kniaznedelin R.A., Smurov A.M. Problemy ekonomiki $i$ upravleniya neftegazovym kompleksom, 2016, no 6, pp. 37-42.

6. Kniaznedelin R.A., Naruzhnyy V.E., Smurov A.M. Gosudarstvennyy zakaz: teoriya, mekhanizm ispolneniya, spetsifika realizatsii $v$ oboronnoy sfere [State order: theory, mechanism of execution, specific features of the defense field]. Ed. by Professor V. A. Plotnikov. SPb., 2009. 159 p.

7. Kozin M.N., Alekseev A.V., Khachatryan G.A. Vestnik Saratovskoy gosudarstvennoy yuridicheskoy akademii, 2009, no 6 (70), pp. 88-91. 
8. Kotliarov I.D. EKO, 2016, no 8, pp. 128-140.

9. Kurbanov A.Kh., Naruzhnyy V.E. Problemy sovremennoy ekonomiki, 2015, no 3, pp. 72-77.

10.Kurbanov A.Kh., Naruzhnyy V.E., Plotnikov V.A. Upravlencheskoe konsultirovanie, 2015, no 10, pp. 101-109.

11. Kurbanov A.Kh., Plotnikov V.A. Izvestiya Yugo-Zapadnogo gosudarstvennogo universiteta, 2011, no 2, pp. 22-30.

12. Kurbanov A.Kh., Plotnikov V.A. Ekonomicheskoe vozrozhdenie Rossii, 2016, no 3, pp. 45-56.

13. Safikhanov A.A., Kozin M.N., Kurbanov T.Kh. Ekonomika i menedzhment system upravleniya, 2016, V. 3.1, no 21, pp. 170-176.

14. Semenikhin A.L. Metodologiya voenno-ekonomicheskogo prognozirovaniya sistemy razvitiya materialnogo obespecheniya [A methodology of military-economic forecast of the system of development of supply]. Dr. Sc. (Military Sciences) Thesis. SPb., 1998. 264 p.

\section{ДАННЫЕ ОБ АВТОРЕ}

Смуров Александр Михайлович, старший научный сотрудник, кандидат экономических наук

Научно-исследовательский институт (военно-системных исследований материально-технического обеспечения Вооружённых Сил Российской Федерации) Военной академии материально-технического обеспечения им. генерала армии А.В. Хрулёва

Наб. Макарова, 8, г. Санкт-Петербург, 199034, Российская Федерачия

a.smurow@mail.ru

\section{DATA ABOUT THE AUTHOR}

Smurov Alexander Mikhailovich, Senior Researcher, Candidate of Economic Sciences

Military Academy of Material and Technical Support named after General of the Army A.V. Khrulev

8, Nab. Makarova, St. Petersburg, 199034, Russian Federation a.smurow@mail.ru 